\title{
Efficacy of a Gas Permeable Contact Lens to Induce Peripheral Myopic Defocus
}

\author{
Jaume Pauné*, Antonio Queiros ${ }^{\dagger}$, Daniela Lopes-Ferreira*, Miguel Faria-Ribeiro*, Lluisa Quevedo ${ }^{\dagger}$, \\ and Jose Manuel Gonzalez-Meijome
}

\begin{abstract}
Purpose. The purpose of this work was to evaluate the potential of a novel custom-designed rigid gas permeable (RGP) contact lens to modify the relative peripheral refractive error in a sample of myopic patients.

Methods. Fifty-two right eyes of 52 myopic patients (mean $[ \pm S D]$ age, $21[ \pm 2]$ years) with spherical refractive errors ranging from -0.75 to -8.00 diopters (D) and refractive astigmatism of $1.00 \mathrm{D}$ or less were fitted with a novel experimental RGP (ExpRGP) lens designed to create myopic defocus in the peripheral retina. A standard RGP (StdRGP) lens was used as a control in the same eye. The relative peripheral refractive error was measured without the lens and with each of two lenses (StdRGP and ExpRGP) using an open-field autorefractometer from 30 degrees nasal to 30 degrees temporal, in 5-degree steps. The effectiveness of the lens design was evaluated as the amount of relative peripheral refractive error difference induced by the ExpRGP compared with no lens and with StdRGP conditions at 30 degrees in the nasal and temporal (averaged) peripheral visual fields.
\end{abstract}

Results. Experimental RGP lens induced a significant change in relative peripheral refractive error compared with the nolens condition (baseline), beyond the 10 degrees of eccentricity to the nasal and temporal side of the visual field $(p<0.05)$. The maximum effect was achieved at 30 degrees. Wearing the ExpRGP lens, $60 \%$ of the eyes had peripheral myopia exceeding $-1.00 \mathrm{D}$, whereas none of the eyes presented with this feature at baseline. There was no significant correlation $(r=0.04 ; p=0.756)$ between the degree of myopia induced at 30 degrees of eccentricity of the visual field with the ExpRGP lens and the baseline refractive error.

Conclusions. Custom-designed RGP contact lenses can generate a significant degree of relative peripheral myopia in myopic patients regardless of their baseline spherical equivalent refractive error.

(Optom Vis Sci 2015;92:00-00)

Key Words: myopia progression, gas permeable contact lens, peripheral refraction

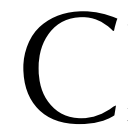

ustomization of the relative peripheral refractive error (RPRE) has become a research goal with the aim of interfering with myopia progression. Treatments to reduce myopia progression in children are available, mainly contact lenses that induce increased vergence of the light entering the eye at an oblique angle (peripheral or off-axis refraction). Orthokeratology provides this effect by reshaping the anterior corneal surface, although this effect is intrinsically dependent on the amount of myopia being corrected. ${ }^{1,2}$

*OD, MSc

${ }^{\dagger} \mathrm{OD}, \mathrm{PhD}$

*OD, PhD, FIACLE

Clinica Telmon, Barcelona, Spain (JP); Clinical and Experimental Optometry Research Lab (CEORLab), Center of Physics, University of Minho, Braga, Portugal (AQ, DL-F, MF-R, JMG-M); and Universidad Politécnica de Cataluña, Terrasa, Spain (LQ).
This effect can also be achieved with center-distance multifocal contact lenses ${ }^{3}$ or spectacles. ${ }^{4}$ The use of contact lens is preferred because they follow the ocular movements and remain centered on the visual axis. Thus, there is a demand for customized contact lenses that can change the pattern of the peripheral refraction, irrespective of the axial refractive error. In this article, the term myopia regulation or myopia control will be used to refer to the decrease in the rate of axial elongation of the eye with different treatments.

We showed in a recent pilot study that experimental rigid and soft contact lenses can induce peripheral myopic defocus irrespective of the patient's baseline axial myopia presented. In that study, the rigid gas permeable (RGP) lens was more effective than the soft lens for creating the peripheral myopic defocus. ${ }^{5}$

The better on-eye stability of the RGP material compared with the soft contact lens materials might explain this outcome. Furthermore, that study showed that a standard aspheric RGP lens 
might also provide some peripheral myopic defocus. Other investigators recently reported a similar observation. ${ }^{6}$

The purpose of the current study was to evaluate the effectiveness of an experimental RGP (ExpRGP) lens to create peripheral myopic defocus in a cohort of myopic patients compared with a standard RGP (StdRGP) design and determine if the axial refraction affects the outcomes.

\section{METHODS}

\section{Subjects and Lenses}

Fifty-two neophyte myopic subjects were enrolled in the study. Measurements were obtained from the right eye only, initially without a lens (baseline), and then with an StdRGP and an ExpRGP lens intended to change the pattern of the RPRE. The two lenses were worn in randomized order on two different days between 9:00 and 11:00 AM and at least 2 hours after awakening.

After receiving an explanation of the nature of the study, each patient signed a consent form before enrollment. The research followed the tenets of the Declaration of Helsinki, and the Ethics Committee for Clinical Research at Clínica Teknon, Barcelona, Spain, reviewed and approved the protocol. The inclusion criteria required that the subjects had up to -8.00 diopters (D) of myopia, had astigmatism less than -1.00 diopter of cylinder (DC), did not have a current ocular disease or injury, were not taking any ocular or systemic medications, and had no contraindications to contact lens wear.

The RGP lenses were made of Boston XO2 (hexafocon B) material with nominal properties of oxygen permeability of 141 barrers, according to the polymer manufacturer (Polymer Technology, MA). The central thicknesses varied depending on the back vertex power of the lenses according to the vertex-corrected spherical equivalent of the noncycloplegic subjective refraction of each eye. The overall diameter was $10.60 \mathrm{~mm}$ for the ExpRGP lens and $10.50 \mathrm{~mm}$ for the StdRGP lens. The base curve radii varied from 7.50 to $8.20 \mathrm{~mm}$ across the subject group, and the same base curve radius was used for both the StdGP and ExpGP lens for each subject. An alignment fit was attempted in all eyes by selecting the base curve radius as a function of the flat keratometric reading and corneal eccentricity.

Experimental RGP lens was designed using Zemax-EE software v.6 (Radiant ZEMAX, WA). Parameters for theoretical eyes were obtained from the study of Atchison ${ }^{7}$ assuming a standard corneal eccentricity of 0.50. Experimental RGP lens has 9 - $\mathrm{mm}$ aspheric front and back optic zones. This design affords $+1.50 \mathrm{D}$ add increase at $2 \mathrm{~mm}$ from center ( $4 \mathrm{~mm}$ chord diameter) corresponding to about 30 degrees of retinal eccentricity and achieving around $+6.5 \mathrm{D}$ at the edge of the optic zone (9 $\mathrm{mm}$ chord diameter) as described in Spanish Patent Application P-201030694.

Standard RGP lenses were commercial lenses PRE AS (Precilens, France) with a central spherical surface followed by a peripheral aspheric surface.

Standard RGP and ExpRGP lenses were fitted according to the topographical information (simK readings measured over the $3 \mathrm{~mm}$ of the central cornea and eccentricity over a chord diameter of $9.0 \mathrm{~mm}$ ). Trial lenses were used to achieve optimal fitting in a prestudy visit. Lenses were ordered based on the vertexed spherical equivalent refraction. Overrefraction was done at the trial visit over the contact lenses and a new lens was ordered if discrepancies greater than $\pm 0.25 \mathrm{D}$ were found. Standard RGP and ExpRGP had the same central distance power.

\section{Peripheral Refraction}

Measurements of the central and peripheral (off-axis) refraction were obtained with an open-field Auto-Refractometer/Keratometer WAM-5500 (Grand Seiko Co, Ltd, Hiroshima, Japan) up to 30 degrees in the nasal and temporal visual field along the horizontal meridian in 5-degree increments. After lens insertion, peripheral refraction was evaluated after cessation of excessive tearing and once the lens was centered in the interblink interval. This took between 5 and 15 minutes after insertion for all eyes evaluated. Each automated refraction measurement was obtained only 1 to 2 seconds after a blink to allow the lens to center and settle. If a blink occurred in the middle of a measurement, the measurement was repeated. To minimize lens decentration during peripheral fixation, head rotation instead of ocular rotation has been used. We used a previously reported method. ${ }^{8,9}$ A laser pointer positioned over the patient's head was oriented toward the primary gaze position. The patient rotated his or her head, avoiding lateral displacement, until the laser pointer reached the given eccentric location while the eyes remained in the primary gaze position.

Descriptive statistics (mean $\pm \mathrm{SD}$ ) were obtained for the refraction vector components $M=\mathrm{Sph}+\mathrm{Cyl} / 2, J_{0}=-\mathrm{Cyl} \cdot \cos (2 \alpha) / 2$, and $J_{45}=-\mathrm{Cyl} \cdot \sin (2 \alpha) / 2$, where Sph, Cyl, and $\alpha$ are the sphere, cylinder, and axis obtained with the autorefractometer, respectively. Relative peripheral refractive error was obtained by subtracting the central refractive error from each eccentric measurement.

\section{Statistical Analysis}

SPSS software package version 19 (SPSS Inc, Chicago, IL) was used for statistical analysis. The Kolmogorov-Smirnov test was applied to assess the normality of data distribution. Considering that we are evaluating different conditions on the same subjects (repeated measures), we used repeated-measures analysis of variance and the Friedman nonparametric test to compare the outcomes between the three conditions (baseline, StdRGP, and ExpRGP values) for normally or non-normally distributed variables, respectively. The Bonferroni post hoc correction for multiple comparisons was applied.

The Spearman rho correlation was applied when normality could not be assumed, and the Pearson correlation was used when normal distribution of data was verified to evaluate the relationship between the RPRE change and baseline refractive error.

For statistical purposes, $\mathrm{p}$ value less than 0.05 was considered significant.

\section{RESULTS}

The mean $( \pm \mathrm{SD})$ central baseline spherical equivalent was -3.22 $( \pm 1.66) \mathrm{D}$ (range, -0.75 to $-8.00 \mathrm{D}$ ) of sphere with less than $1.00 \mathrm{DC}$ of refractive astigmatism in the spectacle plane. The mean $( \pm \mathrm{SD})$ flat keratometric reading was $7.85( \pm 0.25) \mathrm{mm}$.

Statistical analysis showed an interaction between the type of correction and the eccentricity for $M$ and $J_{0}(\mathrm{p}<0.05)$ but not for $J_{45}$. For the most peripheral eccentricities, the $M$ and $J_{0}$ components of refraction became significantly different between the 
Gas Permeable Contact Lens to Induce Peripheral Myopia—Pauné et al. 3
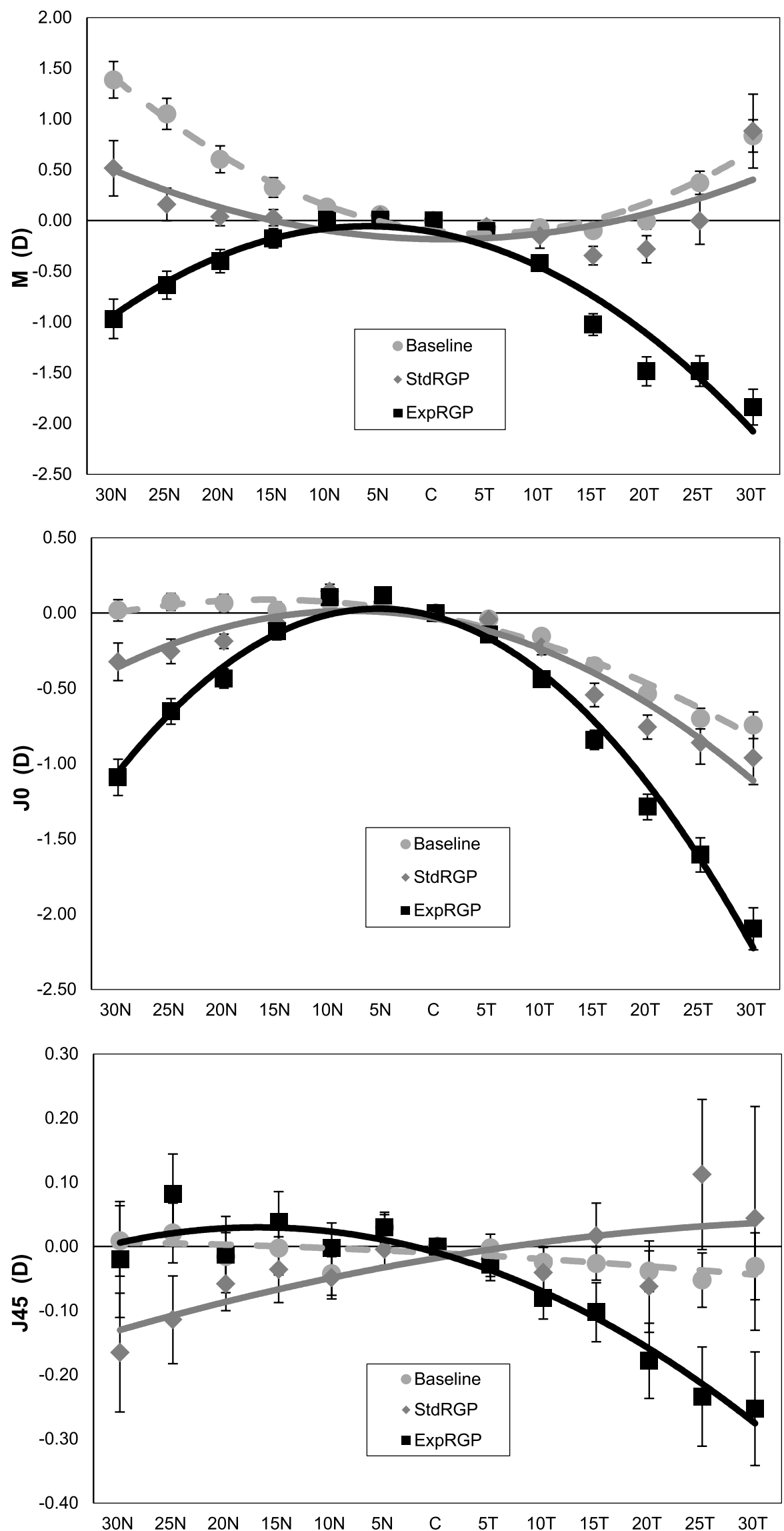

FIGURE 1.

(A) Spherical equivalent component $(M)$ of the RPRE without lenses (baseline) and with StdRGP and ExpRGP lenses. (B, C) Astigmatic component $\left(J_{0}\right.$ and $\left.J_{45}\right)$ of the RPRE without lenses (baseline) and with StdRGP and ExpRGP lenses. Lines represent the second-order polynomial fitting. The error bars represent the SEM. 
experimental conditions evaluated (no lens, ExpRGP, and StdRGP). The comparisons between such conditions at each eccentricity are provided below for $M, J_{0}$, and $J_{45}$ components of refraction.

F1 Fig. 1A shows the RPRE for the $M$ component of refraction in the no-lens condition (baseline) and for the StdRGP and ExpRGP lenses. Statistical analysis showed significant differences in the $M$ component between all examination conditions for the nasal retina beyond 10 degrees (i.e., $\mathrm{p}<0.05$ for 15 degrees and beyond according to analysis of variance) and for the temporal retina beyond 5 degrees (i.e., $\mathrm{p}<0.05$ for 10 degrees and beyond according to the Friedman test). Post hoc tests showed a statistically significant difference between no lens and ExpRGP for all eccentricities beyond 10 degrees in the nasal field and 5 degrees in the temporal field. Standard RGP was significantly different from the no-lens condition for the nasal field beyond 5 degrees of eccentricity and between 15 and 25 degrees of temporal field; however, in this case, the differences were much lower than with the ExpRGP. Differences between StdRGP and ExpRGP were statistically significant beyond 10 degrees of nasal field and beyond 5 degrees of the temporal field.

Fig. 1B, C show the RPRE for $J_{0}$ and $J_{45}$, respectively, with no lens and with StdRGP and ExpRGP lenses. Regarding changes in the $J_{0}$ component, all eccentricities except 15 degrees nasal to 5 degrees temporal showed significant differences when compared with the baseline condition. Post hoc tests showed that ExpRGP presented significantly different RPRE compared with no lens for the nasal eccentricity beyond 10 degrees and for the temporal eccentricity beyond 5 degrees. The same applied for the comparison between StdRGP and ExpRGP. Regarding changes in $J_{45}$, differences between ExpRGP and no lens or between StdRGP and ExpRGP are not statistically significant and are very low.

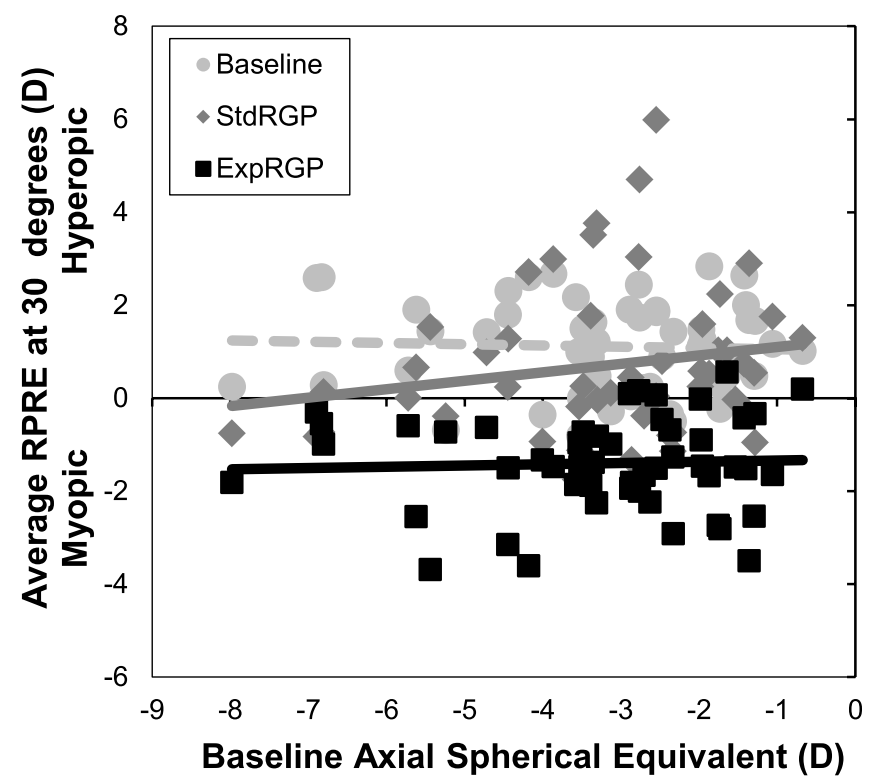

FIGURE 2.

The correlation between the baseline axial refraction and the amount of RPRE (average between 30 degrees in the nasal and temporal visual fields) at baseline without a lens $(y=-0.0275 x+1.0227 ; r=0.05 ; p=0.745$; represented by the light gray circles and dashed line) and the StdRGP lens $(y=0.1815 x+1.2827 ; r=0.19 ; p=0.117$; represented by the dark gray diamonds and dark gray line) and the ExpRGP lens $(y=0.0268 x+1.3164$; $r=0.04 ; p=0.756$; represented by the black squares and black line).

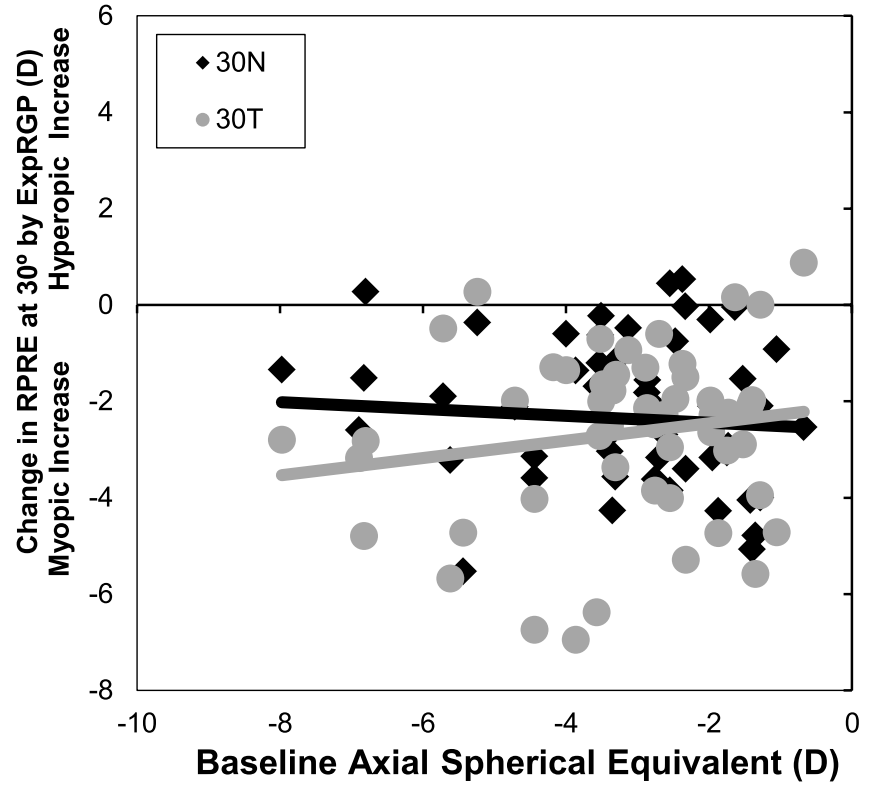

FIGURE 3.

Correlation between the axial refraction at baseline and the amount of change in RPRE achieved with the ExpRGP lens compared with baseline for 30 degrees of eccentricity in the nasal (30 degrees nasal; $y=-0.0715 x-$ $2.585 ; r=0.05 ; p<0.05$; black diamonds, black line) and temporal (30 degrees temporal; $y=0.1802 x-2.0931 ; r=0.16 ; p<0.001$; gray circles, gray line) vistral fields.

Fig. 2 shows the relationship between the spherical equivalent axial refraction without lens and the averaged RPRE at 30 degrees of eccentricity (average of 30 degrees nasal and 30 degrees temporal) for the StdRGP and ExpRGP lenses. Correlations were very weak ( $r=0.05, r=0.19$, and $r=0.04$ for no lens, StdRGP, and ExpRGP, respectively) and nonsignificant. The same results were confirmed when the nasal or temporal RPRE was evaluated independently, instead of averaged.

Fig. 3 shows the relationship between the amount of RPRE in- F3 duced by the ExpRGP lens (ExpRGP minus baseline) at 30 degrees of the temporal and nasal visual field as a function of the baseline axial spherical equivalent. There was negligible or no relationship between central refractive error and RPRE with the ExpRGP lens at 30 degrees nasal $(r=0.05 ; \mathrm{p}<0.05)$ or 30 degrees temporal $(r=0.16$; $\mathrm{p}<0.001)$ eccentricities of the visual field. Despite the statistical significance of the correlation, both showed poor correlation. This means that the RPRE induced was independent of the central refractive error. Overall, in 60\% of the eyes, the ExpRGP lens provided a change in RPRE of at least $-2.00 \mathrm{D}$ of myopia at 30 degrees with a mean $( \pm S D)$ change in this group of $-2.83( \pm 0.56) \mathrm{D}$ (range, -2.01 to $-3.69 \mathrm{D})$.

Fig. 4 shows the averaged nasal and temporal RPRE values for the F4 no-lens condition (baseline) as well as with StdRGP and ExpRGP lenses. Without a lens, only $12 \%$ of the eyes presented some degree of relative peripheral refractive myopia. This increased to $29 \%$ with the StdRGP lens, with most of the eyes showing myopic RPRE below $1.00 \mathrm{D}(21 \%)$ and a few cases (8\%) between 1.00 and $2.00 \mathrm{D}$. With the ExpRGP lens, up to $89 \%$ of the eyes presented some degree of myopic RPRE. In $60 \%$ of the eyes, the ExpRGP lens provided at least $1.00 \mathrm{D}$ of relative peripheral myopia at the 30-degree eccentricity. The total proportion of eyes with RPRE greater than -1.00 was 


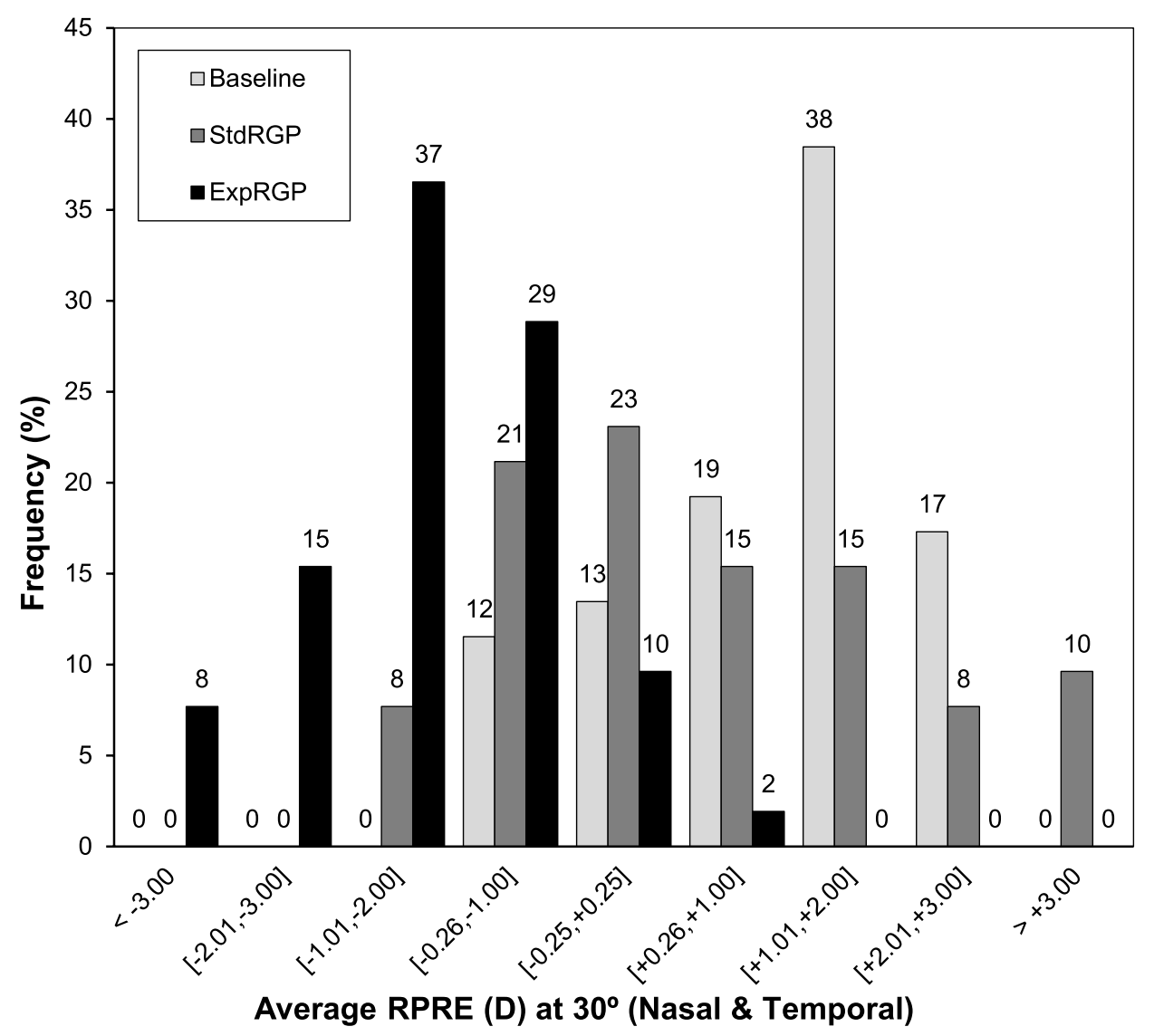

\section{FIGURE 4.}

The proportion of eyes with a given RPRE (average between 30 degrees in the nasal and temporal visual fields) at baseline and with the StdRGP and ExpRGP lenses. Negative values indicate myopic RPRE and positive values indicate hyperopic RPRE.

significantly higher with the ExpRGP compared with StdRGP and no-lens conditions ( $\mathrm{p}<0.05$ for both comparisons, Cochran $Q$ ). When the analysis was conducted for the 30-degree eccentricity in the nasal and temporal fields separately, it was evident that the proportion of eyes with relative peripheral myopia greater than -1.00 was higher in the temporal visual field $(79 \%)$ compared with the nasal visual field (43\%).

F5 Fig. 5 shows the effectiveness of the ExpRGP lens. The following information is depicted in the figure:

- Change induced in RPRE by ExpRGP (ExpRGP minus baseline)

- Change induced in RPRE by StdRGP (StdRGP minus baseline)

- Change induced in RPRE attributable to the special design of the ExpRGP (ExpRGP minus StdRGP)

The ExpRGP lens induced a myopic RPRE change of more than $3.00 \mathrm{D}$ in $37 \%$ of the eyes, between -2.01 and $-3.00 \mathrm{D}$ in $23 \%$, and between -1.01 and $2.00 \mathrm{D}$ in $25 \%$. This makes up to $85 \%$ of the eyes with a difference of at least $-1.00 \mathrm{D}$ of myopic RPRE compared with the no-lens condition. Conversely, the StdRGP lens induced such differences in only 6, 8, and 19\%, respectively (total, 33\%). When the analysis was conducted for the 30-degree eccentricity in the nasal and temporal fields separately, it was evident that the ExpRGP lens provided larger changes in myopic defocus in the temporal field compared with the nasal field. The total amount of eyes presenting changes in RPRE greater than -1.00 of myopia was $45 \%$ when the nasal field was considered and $66 \%$ when the temporal field was considered. Those presenting changes of $-3.00 \mathrm{D}$ or higher were $15 \%$ when the nasal field was considered and $29 \%$ when the temporal field was considered.

\section{DISCUSSION}

Rigid gas permeable materials are excellent platforms for contact lens design because of their optical properties and resistance to flexure forces while on the eye. They are considered safe because they are associated with low risks of infection and other adverse events. ${ }^{10}$ Despite this, they represent a small part of the contact lens market because of their initial discomfort and the need for longer periods of adaptation compared with soft contact lenses. ${ }^{11}$

The current study showed for the first time that a custom RGP lens might effectively induce relative peripheral myopic defocus across a wide range of values of axial myopia. Contrary to the relationship between induced peripheral myopic defocus and central refractive error in orthokeratology treatments, ${ }^{1}$ the amount of myopia induced in the periphery of the visual field with the lens evaluated in the present study is not limited by, or related to, the axial refraction.

Queiros et al. ${ }^{1}$ reported that the relationship between the amount of myopia corrected in the corneal center and the amount of peripheral myopia induced after treatment follows an almost 1:1 pattern, such that for $1.00 \mathrm{D}$ of myopia to be compensated for, there 


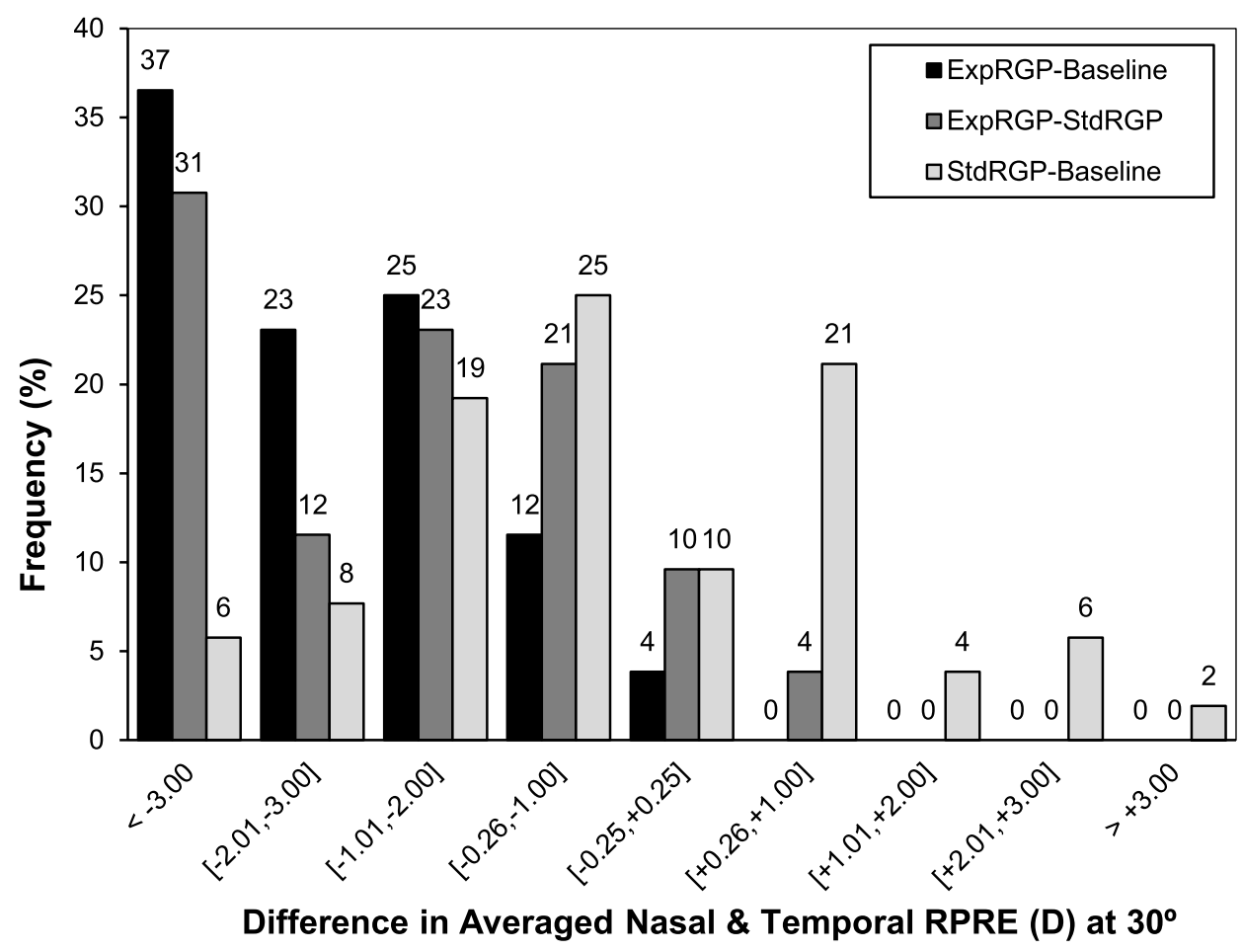

\section{FIGURE 5.}

The proportion of eyes with a given change in RPRE (average between 30 degrees in the nasal and temporal visual fields) for the paired comparisons between baseline, the StdRGP lens, and the ExpRGP lens. Negative values indicate myopic RPRE and positive values indicate hyperopic RPRE.

is the potential to induce $1.00 \mathrm{D}$ of RPRE at 30 degrees in the extremes of the horizontal visual field. Cho et al. ${ }^{12}$ showed that for myopes with less than $2.00 \mathrm{D}$ of myopia, the progression of the axial elongation was very similar to subjects wearing spectacles. Kakita et al. ${ }^{13}$ found a similar trend in Japanese myopic children, but this result was not observed in two other recently published studies from Spain ${ }^{14,15}$ and Hong Kong. ${ }^{16}$

Soft contact lenses currently in use to address myopia regulation incorporate treatment zones of $+2.00 \mathrm{D}$ including multifocal center-distance, peripheral gradient, ${ }^{17}$ and dual-focus lens designs. ${ }^{18}$ The mechanism underlying myopia control through manipulation of the peripheral optics is not fully understood; hence, it is not possible to establish the minimal effective level of peripheral myopia to reduce myopia progression. However, our results showed that if at least 2.00 D of relative peripheral myopic defocus might effectively be used to manipulate the relative peripheral myopic defocus, such an effect could be achieved in $60 \%$ of eyes evaluated in the current study.

Although soft contact lenses might result in faster adaptation associated with better comfort and centration with the pupil ${ }^{19}$ RGPs appear to offer the best platform to create a given optical design and maintain such properties while being worn on the eye. Such an effect cannot be provided in the same way using soft contact lens materials. In our previous study, Proclear center-distance multifocal contact lenses with $+4.00 \mathrm{D}$ of add did not show significantly different peripheral myopic defocus compared with the +3.00 -D add lenses; similarly, $+1.00 \mathrm{D}$ of add did not show any measurable benefit compared with the naked eye. ${ }^{3}$ We reported a similar effect with the same lens in myopic subjects. ${ }^{20}$ Recently, Ticak and Walline ${ }^{21}$ did not find a significant peripheral myopic defocus effect with the Proclear multifocal lenses compared with the effect produced by orthokeratology. However, they used an add power of $+2.00 \mathrm{D}$, which, in our studies, showed only a slight peripheral myopic defocus effect compared with the more effective $+3.00 \mathrm{D}$ add. This means that a given increase in the peripheral power addition on a soft contact lens material does not necessarily increase the effect in the same proportion when measured by peripheral refraction. This has been confirmed recently in a pilot study that compared the peripheral refraction between the current ExpRGP design and the same design manufactured in a hydrogel material. ${ }^{5}$ In contrast, it appears that the effect of the RGP lens might be stronger (average change in RPRE, $-2.00 \mathrm{D}$ ) than the one intended to be reproduced $(-1.50 \mathrm{D}$ as expected from the lens design at 30 degrees). In the present work, it has been found that the change induced in the RPRE can be greater than 2.00 or even $3.00 \mathrm{D}$ in a significant proportion of eyes with the ExpRGP lens. This would not be expected as the lens is designed to induce up to $1.5 \mathrm{D}$ of myopic defocus. To find an explanation to the higher spherical equivalent obtained compared with the theoretical peripheral myopic defocus induced by the lens design, we have to consider the astigmatism induced by the oblique incidence of light. When computed as spherical equivalent, the astigmatism induced by the optical surfaces for oblique incidence of light into the eye contributes to an overall increase in the myopic defocus that is beyond the change expected attributed solely to the increase in spherical power by the design of the lens. Additionally, the use of an autorefractor, which averages refraction over a $2.3-\mathrm{mm}$ diameter measurement ring, is likely to give highly myopic readings as the power add continues increasing up to the edge of the optic zone as explained in METHODS.

Ticak and Walline ${ }^{21}$ suggested in their recent study that more accurate instruments are needed to measure the power at discrete points in lenses with strong changes in power across their surface while the lenses are on the eye to better understand the refractive 
effect of orthokeratology treatment and multifocal contact lenses in the context of myopia progression. However, previous studies have shown similar effects of the Proclear center-distance lens using an aberrometer that measures the full pupillary area. ${ }^{22}$ Furthermore, because previous studies of orthokeratology and other lenses also have been conducted with the Grand Seiko or the Shin-Nippon instruments, the current results were comparable to those previously reported.

Our results show that the defocus effect might be different at different retinal areas. As an example, although more than $80 \%$ of the eyes will experience a change in RPRE of at least $-1.00 \mathrm{D}$, this amount reduces to $45 \%$ when we consider the nasal field. For 29\% of the subjects, the amount of the RPRE with the ExpRGP lens ranged from 0.25 to $1.00 \mathrm{D}$ of myopia. The peripheral refraction and probably the retinal shape are not the same between the horizontal and vertical meridians, ${ }^{21,23}$ not even between the nasal and temporal sides. ${ }^{24}$ Our results show that with the present lenses, higher myopic defocus is usually achieved in the temporal visual field compared with the nasal visual field. We cannot justify these outcomes by the decentration of the lens with gaze changes. However, slight temporal decentration of the RGP lenses is usually observed, even when the lens remains within the accepted levels of centration $(0.50 \mathrm{~mm}){ }^{25}$ In the present study, lens centration was improved using the large-diameter lens (10.60 mm for the ExpRGP lens and $10.50 \mathrm{~mm}$ for the StdRGP lens). This is critical when fitting RGP lenses to maximize the symmetry in the myopic defocus induced around the foveal area. To maximize centration during the peripheral measurements, head turn instead of eye turn was used to fixate peripheral targets.

Results presented in this study show that the asymmetry between refraction in the nasal and temporal retinal eccentricity was approximately constant without and with lenses. Any significant decentration of the lens during measurements would result in reduction or exaggeration of the asymmetry found. This asymmetry in baseline data has been previously documented. ${ }^{8,20,26,27}$ This might be related to the asymmetry in the nasal and temporal eye length and corresponding posterior retinal contour in myopia. ${ }^{24,28}$ It could be also related to the measuring technique as we centered our measurements on the pupillary center. Asymmetries between nasal and temporal corneal curvature could also account for some differences. Kwok et al. ${ }^{8}$ found that asymmetries between nasal and temporal peripheral refractive error would be minimized if measurements were referred to the optical axis of the eye instead of the foveal axis. Overall, it can be concluded that lens decentration does not contribute to a change in the asymmetry of the nasal and temporal refraction and the factors previously mentioned should be considered.

Considering that treatments attempting to slow myopia progression should be more effective in younger children, safety becomes a major concern. Different authors have reported that RGP lenses worn overnight during orthokeratology treatment are well tolerated by children, ${ }^{13,14,29}$ which is probably related to the fast adaptation to this modality. ${ }^{30}$ Children also have been shown to successfully adapt to RGP lenses on a daily wear basis in different clinical trials. ${ }^{19,31}$ This makes RGP lenses a viable option for controlling myopia progression even if they have to be worn on a daily wear basis. One relevant aspect is the higher dropout rate that might be present with RGP compared with soft contact lenses. In a randomized clinical trial addressing the potential efficacy of conventional RGP lenses for myopia progression, Walline et al. ${ }^{31}$ reported a $70 \%$ retention rate in the RGP arm compared with $93 \%$ in the soft contact lens control arm. Although the tolerance must be a limitation for patients who previously wore soft contact lenses, this might not be the case for most young children wearing lenses for the first time to control myopia. Finally, the data summarized in the Tear Film \& Ocular Surface Society Interna- $\mathbf{A Q 1}$ tional Workshop on Contact Lens Discomfort showed a trend for RGP lenses with larger diameters being more comfortable. ${ }^{32,33}$ As in the present study, RGP lenses intended for myopia regulation should be manufactured in larger-than-average diameters to improve centration and provide a sufficiently large treatment zone over the larger pupil areas of children. Furthermore, to overcome the shortterm discomfort issues with corneal RGP lenses, other strategies might be considered in the future, such as large-diameter scleral or hybrid lenses. These lenses can also provide more lens stability and centration against misalignments related to ocular rotation and blinking.

In summary, the present study showed that RGP lenses can be custom designed to provide myopic RPRE in most of the myopic eyes irrespective of the degree of axial myopia within the range from -1.00 to $-8.00 \mathrm{D}$ of spherical equivalent included in this study. Comfort-related aspects of this modality compared with soft contact lenses need to be considered. Finally, although the efficacy of this device in providing myopic RPRE has been shown, the efficacy to inhibit myopia progression still needs to be investigated with the appropriate clinical trial.

\section{ACKNOWLEDGMENTS}

Jaume Paune has proprietary and financial interests in the manufacturing and distribution of lenses evaluated in this study. The remaining authors declare that they do not have any proprietary or financial interest in any of the materials mentioned in this article. This work was partially funded by Fundação para Ciência e Tecnologia, Portugal, Projects: PTDC/SAU-BEB/ 098392/2008 and PTDC/SAU-BEB/098391/2008.

Received March 5, 2013; accepted March 4, 2015.

\section{REFERENCES}

1. Queiros A, Gonzalez-Meijome JM, Jorge J, Villa-Collar C, Gutierrez AR. Peripheral refraction in myopic patients after orthokeratology. Optom Vis Sci 2010;87:323-9.

2. Charman WN, Mountford J, Atchison DA, Markwell EL. Peripheral refraction in orthokeratology patients. Optom Vis Sci 2006;83: 641-8.

3. Lopes-Ferreira D, Ribeiro C, Maia R, Garcia-Porta N, Queiros A, Villa-Collar C, Gonzalez-Meijome JM. Peripheral myopization using a dominant design multifocal contact lens. J Optom 2011;4: $14-21$.

4. Sankaridurg P, Donovan L, Varnas S, Ho A, Chen X, Martinez A, Fisher S, Lin Z, Smith EL, 3rd, Ge J, Holden B. Spectacle lenses designed to reduce progression of myopia: 12-month results. Optom Vis Sci 2010;87:631-41.

5. Paune J, Queiros A, Quevedo L, Neves H, Lopes-Ferreira D, Gonzalez-Meijome JM. Peripheral myopization and visual performance with experimental rigid gas permeable and soft contact lens design. Cont Lens Anterior Eye 2014;37:455-60.

6. Shen J, Clark CA, Soni PS, Thibos LN. Peripheral refraction with and without contact lens correction. Optom Vis Sci 2010;87:642-55.

7. Atchison DA. Optical models for human myopic eyes. Vision Res 2006;46:2236-50. 
8 Gas Permeable Contact Lens to Induce Peripheral Myopia-Pauné et al.

8. Kwok E, Patel B, Backhouse S, Phillips JR. Peripheral refraction in high myopia with spherical soft contact lenses. Optom Vis Sci 2012;89:263-70.

9. Radhakrishnan H, Charman WN. Peripheral refraction measurement: does it matter if one turns the eye or the head? Ophthalmic Physiol Opt 2008;28:73-82.

10. Morgan PB, Efron N, Hill EA, Raynor MK, Whiting MA, Tullo AB. Incidence of keratitis of varying severity among contact lens wearers. Br J Ophthalmol 2005;89:430-6.

11. Fonn D, Gauthier CA, Pritchard N. Patient preferences and comparative ocular responses to rigid and soft contact lenses. Optom Vis Sci 1995;72:857-63.

12. Cho P, Cheung SW, Edwards M. The longitudinal orthokeratology research in children (LORIC) in Hong Kong: a pilot study on refractive changes and myopic control. Curr Eye Res 2005;30:71-80.

13. Kakita T, Hiraoka T, Oshika T. Influence of overnight orthokeratology on axial elongation in childhood myopia. Invest Ophthalmol Vis Sci 2011;52:2170-4.

14. Santodomingo-Rubido J, Villa-Collar C, Gilmartin B, GutiérrezOrtega R. Myopia control with orthokeratology contact lenses in Spain (MCOS): study design and general baseline characteristics. J Optom 2009;2:215-22.

15. Santodomingo-Rubido J, Villa-Collar C, Gilmartin B, GutierrezOrtega R. Myopia control with orthokeratology contact lenses in Spain: refractive and biometric changes. Invest Ophthalmol Vis Sci 2012;53:5060-5.

16. Cho P, Cheung SW. Retardation of myopia in Orthokeratology (ROMIO) study: a 2-year randomized clinical trial. Invest Ophthalmol Vis Sci 2012;53:7077-85.

17. Sankaridurg P, Holden B, Smith E, 3rd, Naduvilath T, Chen X, de la Jara PL, Martinez A, Kwan J, Ho A, Frick K, Ge J. Decrease in rate of myopia progression with a contact lens designed to reduce relative peripheral hyperopia: one-year results. Invest Ophthalmol Vis Sci 2011;52:9362-7.

18. Anstice NS, Phillips JR. Effect of dual-focus soft contact lens wear on axial myopia progression in children. Ophthalmology 2011;118: 1152-61.

19. Jones-Jordan LA, Walline JJ, Mutti DO, Rah MJ, Nichols KK, Nichols JJ, Zadnik K. Gas permeable and soft contact lens wear in children. Optom Vis Sci 2010;87:414-20.

20. Lopes-Ferreira D, Ribeiro C, Neves H, Faria-Ribeiro M, Queiros A, Villa-Collar C, Jorge J, Gonzalez-Meijome JM. Peripheral refraction with dominant design multifocal contact lenses in young myopes. J Optom 2013;6:85-94.

21. Ticak A, Walline JJ. Peripheral optics with bifocal soft and corneal reshaping contact lenses. Optom Vis Sci 2013;90:3-8.
22. Rosen R, Jaeken B, Lindskoog Petterson A, Artal P, Unsbo P, Lundstrom L. Evaluating the peripheral optical effect of multifocal contact lenses. Ophthalmic Physiol Opt 2012;32:527-34.

23. Atchison DA, Pritchard N, Schmid KL. Peripheral refraction along the horizontal and vertical visual fields in myopia. Vision Res 2006;46:1450-8.

24. Faria-Ribeiro M, Queiros A, Lopes-Ferreira D, Jorge J, GonzalezMeijome JM. Peripheral refraction and retinal contour in stable and progressive myopia. Optom Vis Sci 2013;90:9-15.

25. Wolffsohn JS, Hunt OA, Basra AK. Simplified recording of soft contact lens fit. Cont Lens Anterior Eye 2009;32:37-42.

26. Lopes-Ferreira DP, Neves HI, Faria-Ribeiro M, Queiros A, Fernandes PR, Gonzalez-Meijome JM. Peripheral refraction with eye and head rotation with contact lenses. Cont Lens Anterior Eye, 2014.

27. Lin Z, Martinez A, Chen X, Li L, Sankaridurg P, Holden BA, Ge J. Peripheral defocus with single-vision spectacle lenses in myopic children. Optom Vis Sci 2010;87:4-9.

28. Logan NS, Gilmartin B, Wildsoet CF, Dunne MC. Posterior retinal contour in adult human anisomyopia. Invest Ophthalmol Vis Sci 2004;45:2152-62.

29. Walline JJ, Jones LA, Sinnott LT. Corneal reshaping and myopia progression. Br J Ophthalmol 2009;93:1181-5.

30. Gonzalez-Meijome JM, Carracedo G, Gonzalez-Perez J, Peral A, Jorge J, Peixoto-De-Matos SC. Comfort and vision scores at insertion and removal during 1 month of wear of Paragon CRT for corneal reshaping. Eye Contact Lens 2011;37:302-6.

31. Walline JJ, Jones LA, Mutti DO, Zadnik K. A randomized trial of the effects of rigid contact lenses on myopia progression. Arch Ophthalmol 2004;122:1760-6.

32. Sorbara L, Mueller K. Effect of lens diameter on lens performance and initial comfort of two types of GP lenses for keratoconus: a pilot study. J Optom 2011;4:22-9.

33. Jones L, Brennan NA, Gonzalez-Meijome J, Lally J, MaldonadoCodina C, Schmidt TA, Subbaraman L, Young G, Nichols JJ. The TFOS International Workshop on Contact Lens Discomfort: report of the contact lens materials, design, and care subcommittee. Invest Ophthalmol Vis Sci 2013;54:TFOS37-70.

José Manuel González-Méijome Clinical and Experimental Optometry Research Lab Department of Physics (Optometry) University of Minho 4710-057 Braga Portugal e-mail: jgmeijome@fisica.uminho.pt 


\section{AUTHOR QUERY}

AUTHOR PLEASE ANSWER QUERY

AQ1 = "TFOS CLD workshop" was expanded as "Tear Film \& Ocular Surface Society International Workshop on Contact Lens Discomfort". Is this correct?

END OF AUTHOR QUERY 